\title{
The Angular Momenta Dipole Moments and Gyromagnetic Ratios of the Electron and the Proton
}

\section{A. Georgiou}

School of Physics Astronomy and Mathematics, University of Hertfordshire, Hatfield, UK Email: a.georgiou@herts.ac.uk

Received 3 June 2014; revised 1 July 2014; accepted 24 July 2014

Copyright (C) 2014 by author and Scientific Research Publishing Inc.

This work is licensed under the Creative Commons Attribution International License (CC BY). http://creativecommons.org/licenses/by/4.0/

c) (i) Open Access

\begin{abstract}
We had previously obtained analytical formulae for the dipole moments and angular momenta of rotating spherical bodies. The resulting formulae were applied to the Sun, the star 78 Virginis and the Earth. The agreement of the theoretical formulae with the actual real situations is indeed remarkable. In this note we apply the same formulae to the electron and the proton, using the classical values of the radii, so no quantum mechanical treatment is considered.
\end{abstract}

Keywords

Angular Momenta, Dipole Moments, Electron, Proton

\section{Introduction}

The purpose of this work is to obtain analytical formulae for the dipole moments and angular momenta of the electron and the proton. For this purpose, exact solutions of the Einstein-Maxwell field equations for volume distributions of rotating charged matter are required. There are difficulties in obtaining such solutions, but these were successfully treated in detail in [1]. In addition, there are uncertainties regarding the radii of these particles, resulting in some differences between the calculated numerical values and the accepted values of the dipole moments.

\section{The Electron}

The mass $m$, classical radius $a$, the electric charge $e$ of the electron and the vacuum speed of light, are: 


$$
\begin{aligned}
& m=9.109534 \times 10^{-28} \mathrm{~g} \\
& a=2.81794029 \times 10^{-13} \mathrm{~cm} \\
& e=-4.80320427 \times 10^{-10} \mathrm{esu} \\
& c=2.99792458 \times 10^{10} \mathrm{~ms}^{-1} .
\end{aligned}
$$

The following ratios are required:

$$
\begin{aligned}
& m / a=3.23263879 \times 10^{-15} \\
& G / c^{2}=7.4242465 \times 10^{-29} \\
& G / c^{4}=8.26058829 \times 10^{-50} \\
& \sqrt{G} / c=8.61640674 \times 10^{-15} .
\end{aligned}
$$

If the mass is $m$, then there is an electric charge $m \sqrt{G}$ associated with $m$ [1]. Thus, if there is an additional electric charge $q$, the total charge is $q+m \sqrt{G}$ esu. The value of $m \sqrt{G}$ is only $2.353114478 \times 10^{-31}$ and so it is negligible compared to the value of $e$ in (1). If $q=e$, we may therefore write for the charge $q$ and its square $q^{2}$,

$$
\begin{aligned}
& q=-4.803206799 \times 10^{-10}=e \\
& q^{2}=e^{2}=2.30711374 \times 10^{-19} .
\end{aligned}
$$

In the Reissner-Nordstrom solution [2], the coefficient of $\mathrm{d} t^{2}$ in the metric, has the form given by

$$
F_{R N}=1-\frac{2 G m}{c^{2} r}+\frac{G q^{2}}{c^{4} r^{2}}
$$

with $q^{2}$ given by the second of (3).

The coefficient of $\mathrm{d} t^{2}$ in the Kerr-Newman metric, is

$$
F_{K N}=\frac{\Delta-j^{2} \sin ^{2} \theta}{r^{2}+j^{2} \cos ^{2} \theta}
$$

with

$$
\Delta=j^{2}+r^{2} F_{R N}
$$

Equation (5) then, becomes

$$
F_{K N}=\left\{1+\frac{-\frac{2 G m r}{c^{2}}+\frac{G q^{2}}{c^{4}}}{r^{2}+j^{2} \cos ^{2} \theta}\right\}
$$

where $j$ is the angular momentum per unit mass; see Equations (18)-(20) of [2]. On $\theta=\pi / 2, \cos \theta=0$ and so on $r=a \quad$ Equation (7) becomes

$$
F_{K N}=1-\frac{2 G m}{c^{2} a}+\frac{G q^{2}}{c^{4} a^{2}}
$$

which is the same as Equation (2) of [2] (Note that in reference [2] $f$ is used where we use $F$ ). It follows that instead of Equations (7)-(8) of [1], we have for $r=a$

$$
\lambda^{2}=\frac{2 G m}{c^{2} a}-\frac{G q^{2}}{c^{4} a^{2}}
$$

In accordance with the results of [1], the dipole moment $P$, total angular momentum $J$ and gyromagnetic ratio are: 


$$
\begin{aligned}
& P=\frac{3}{2} b^{2} a^{2} \lambda\left(1-\lambda^{2}\right) \frac{c^{2}}{\sqrt{G}} \\
& J=\frac{3}{2} b a^{2} \lambda \frac{c^{3}}{G} \\
& \frac{P}{J}=b\left(1-\lambda^{2}\right) \frac{\sqrt{G}}{c}
\end{aligned}
$$

where

$$
b=\frac{1}{C(a)} C(a)=1+\frac{G m}{c^{2} a}-\frac{G q^{2}}{2 c^{4} a^{2}} .
$$

The values in (2) and (3) give for $\lambda^{2}$ in (9) and $\lambda$

$$
\begin{aligned}
& \lambda^{2}=2.399952881 \times 10^{-43} \\
& \lambda=4.898931395 \times 10^{-22}
\end{aligned}
$$

Equations (10) then give $P=2.030255524 \times 10^{-22} \mathrm{erg} /$ Gauss , or

$$
P=0.2030255524 \times 10^{-24} \mathrm{~J} / \mathrm{T} \text {. }
$$

The accepted value $P_{a}$ is

$$
P_{a}=9.284764 \times 10^{-24} \mathrm{~J} / \mathrm{T} .
$$

From the second of (10), we obtain for $J$

$$
J=2.356267042 \times 10^{-8} .
$$

The gyromagnetic ratio is therefore,

$$
P / J=8.61640674 \times 10^{-15}
$$

and this is precisely the value of $\sqrt{G} / c$.

It must be noted that there are various different values for the radius of the classical electron. The value of $P$ found is smaller than the accepted value and it depends on which radius we choose. Thus, if we use a radius with numerical value

$$
a=20 \times 10^{-13} \mathrm{~cm}
$$

which is 7 times larger than the classical radius of the electron and repeat the calculations, we find

$$
P=5.234278 \times 10^{-24} \mathrm{~J} / \mathrm{T} \text {. }
$$

This is nearer the accepted value in (14).

It was established by Dehmelt [3] that the upper limit for the electron radius is $10^{-17} \mathrm{~cm}$.

This simply implies that if $a_{e}$ is the electronic radius, we must have $a_{e}<10^{-17}$. The values of the radii we used, including the "classical radius of the electron", are much larger than this.

\section{The Proton}

The mass, radius, electric charge and mass to radius ratio of the proton, are respectively:

$$
\begin{aligned}
& m_{p}=1.672621637 \times 10^{-24} \mathrm{~g} \\
& a_{p}=0.84 \times 10^{-13} \mathrm{~cm} . \\
& -e=4.80320427 \times 10^{-10} \mathrm{esu} \\
& m_{p} / a_{p}=1.991216235 \times 10^{-11} .
\end{aligned}
$$

If there is an additional electric charge $q$, the total charge will be $q+m \sqrt{G}$ esu. The value of $m \sqrt{G}$ is only $4.320605412 \times 10^{-28}$ esu and so it is negligible compared to the value of $e$ in (1). If $q=e$, we may 
therefore write for the total charge $q$ and its square $q^{2}$,

$$
\begin{aligned}
& q=4.803206799 \times 10^{-10}=-e \\
& q^{2}=e^{2}=2.30711374 \times 10^{-19} .
\end{aligned}
$$

The same Formulae (12) for $\lambda^{2}$ and (10) for $P, J$ and $P / J$ are valid, but with the proton parameters in Equations (19) instead of the electron ones. We find

$$
\begin{aligned}
& \lambda^{2}=2.950901603 \times 10^{-39} \\
& \lambda=5.432220175 \times 10^{-20} \\
& P=2.004568264 \times 10^{-24} \\
& J=23.26455011 \times 10^{-8} \\
& P / J=8.61640674 \times 10^{-15}=\sqrt{G} / c
\end{aligned}
$$

We note that the gyromagnetic ratio found in the $5^{\text {th }}$ of Equations (21), is precisely the value of $\sqrt{G} / c$.

In the case of the proton the value of $P$ found is larger than the accepted value, which is $0.014 \times 10^{-24} \mathrm{~J} / \mathrm{T}$.

\section{Conclusion}

We have obtained the dipole moments angular momenta and gyromagnetic ratios of the electron and the proton using the analytical formulae developed in [1]. In the case of the electron, the value of $P$ found is smaller than the accepted value, but in the proton case it is larger. If Dehmelt's [3] deductions are valid, a complete reevaluation is necessary using our analytical Formulae (10) to find $P, J$ and $P / J$. It is not possible to do this, because Dehmelt, does not give any definite values for the radii; he only states that the electron radius $a_{e}$ should satisfy the inequality $a_{e}<10^{-17}$. But in any case, the purpose of the calculations here, is to see if the values of the known classical electron and proton radii, give the expected values of $P, J$ and $P / J$.

\section{References}

[1] Georgiou, A. (2012) Journal of Modern Physics, 3, 1301-1310.

[2] Blinder, S.M. (2003) Dirac’s Electron via General Relativity. PACS No: 03.50.De, 14.60.C.

[3] Dehmelt, H. (1988) Physical Scripta, T22, 102-110. http://dx.doi.org/10.1088/0031-8949/1988/T22/016 
Scientific Research Publishing (SCIRP) is one of the largest Open Access journal publishers. It is currently publishing more than 200 open access, online, peer-reviewed journals covering a wide range of academic disciplines. SCIRP serves the worldwide academic communities and contributes to the progress and application of science with its publication.

Other selected journals from SCIRP are listed as below. Submit your manuscript to us via either submit@scirp.org or Online Submission Portal.
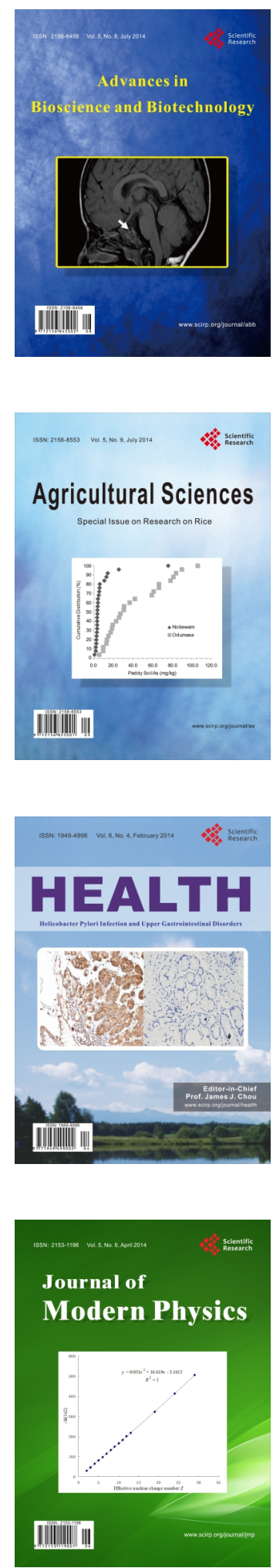
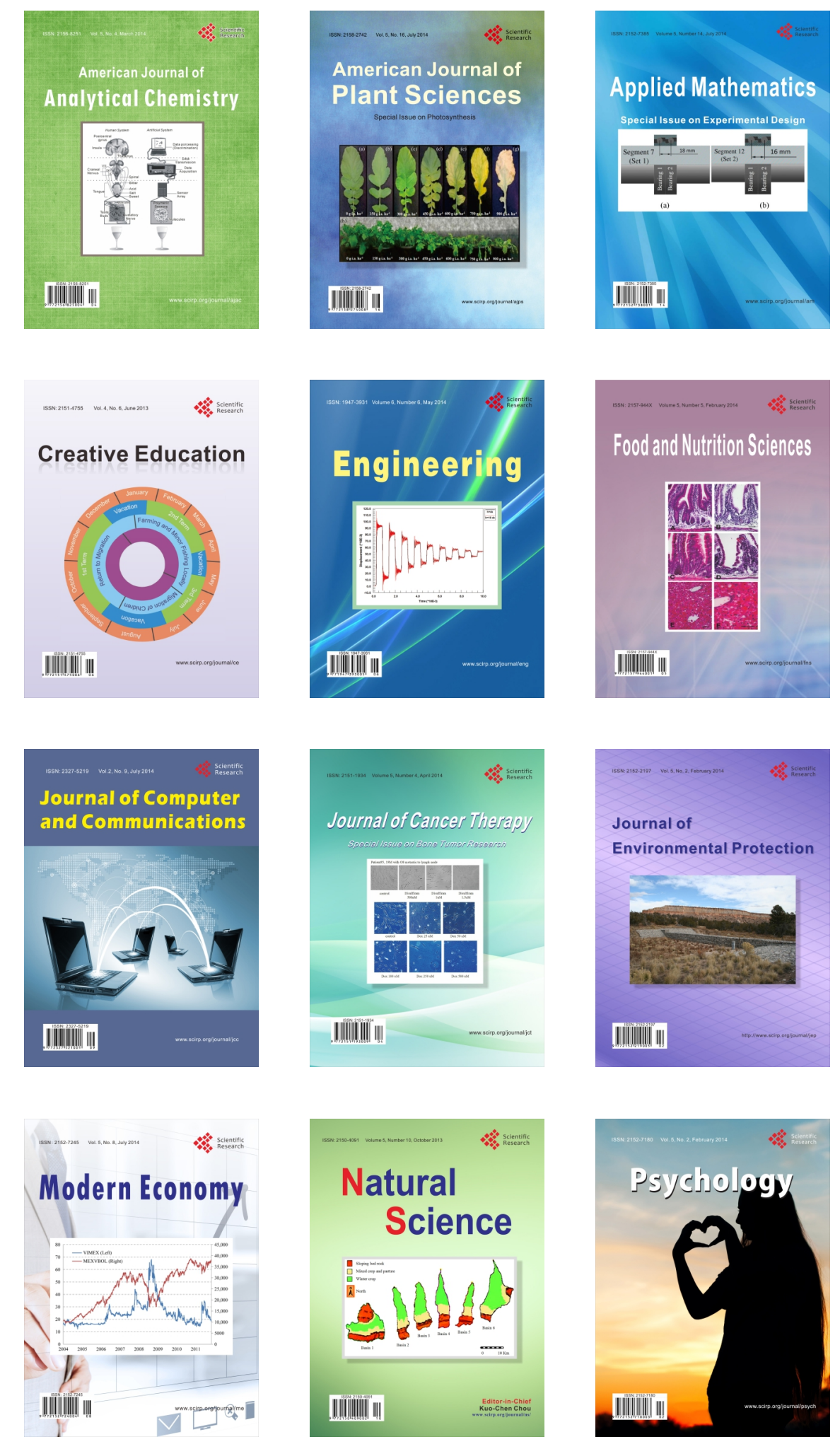